\title{
Corrigendum to "Zanthoxylum ailanthoides Suppresses Oleic Acid-Induced Lipid Accumulation through an Activation of LKB1/AMPK Pathway in HepG2 Cells"
}

\author{
Eun-Bin Kwon, ${ }^{1,2}$ Myung-Ji Kang, ${ }^{1,2}$ Soo-Yeon Kim, ${ }^{1,2}$ Yong-Moon Lee, ${ }^{2}$ \\ Mi-Kyeong Lee, ${ }^{2}$ Heung Joo Yuk, ${ }^{1}$ Hyung Won Ryu, ${ }^{1}$ Su Ui Lee, ${ }^{1}$ Sei-Ryang Oh (D), \\ Dong-Oh Moon $\mathbb{D},{ }^{3}$ Hyun-Sun Lee $\mathbb{D},{ }^{1}$ and Mun-Ock Kim $\mathbb{D}^{1}$ \\ ${ }^{1}$ Korea Research Institute of Bioscience and Biotechnology (KRIBB), Cheongju, Chungbuk 28116, Republic of Korea \\ ${ }^{2}$ College of Pharmacy, Chungbuk National University, Cheongju, Chungbuk 28644, Republic of Korea \\ ${ }^{3}$ Department of Biology Education, Daegu University, Gyeongsan-si, Gyeongsangbuk 38453, Republic of Korea \\ Correspondence should be addressed to Mun-Ock Kim; mokim@kribb.re.kr
}

Received 1 July 2019; Accepted 2 July 2019; Published 14 July 2019

Copyright (C) 2019 Eun-Bin Kwon et al. This is an open access article distributed under the Creative Commons Attribution License, which permits unrestricted use, distribution, and reproduction in any medium, provided the original work is properly cited.

In the article titled "Zanthoxylum ailanthoides Suppresses Oleic Acid-Induced Lipid Accumulation through an Activation of LKB1/AMPK Pathway in HepG2 Cells" [1], there was figure duplication in Figure 4, where the second panel (AMPK) in Figure 4(b) is the same as the last panel (Actin) in Figure 4(d).

The authors explained that, at the beginning, Figures 4(b) and 4(d) were one figure consisting of p-AMPK band (first panel in Figure 4(b)) and Actin band (last panel in Figure 4(d)). AMPK band (the second panel in Figure 4(b)) did not exist, as it needed to be confirmed through a Western blot experiment. Then, the Western blot results of p-LKB 1 and LKB 1 bands, performed under the same experimental conditions, were added. At the same time, due to a mix-up, the AMPK band (the second panel in Figure 4(b)) and the Actin band (last panel in Figure 4(d)) were duplicated. They clarified they did not recognize this duplication and the figure was then subdivided into Figures 4(b) and 4(d) and Actin band (the last panel in Figure 4(b)) was added. Therefore, Figure 4 should be corrected as shown below. 

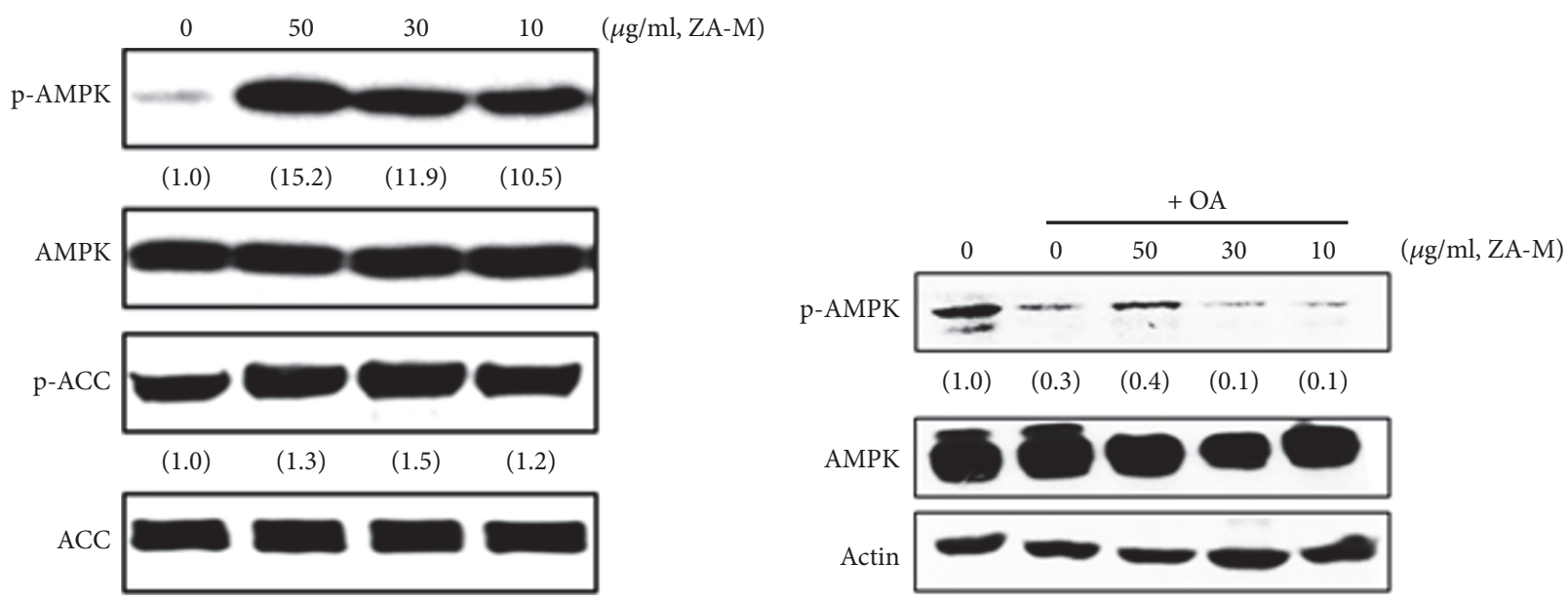

(a)

(b)

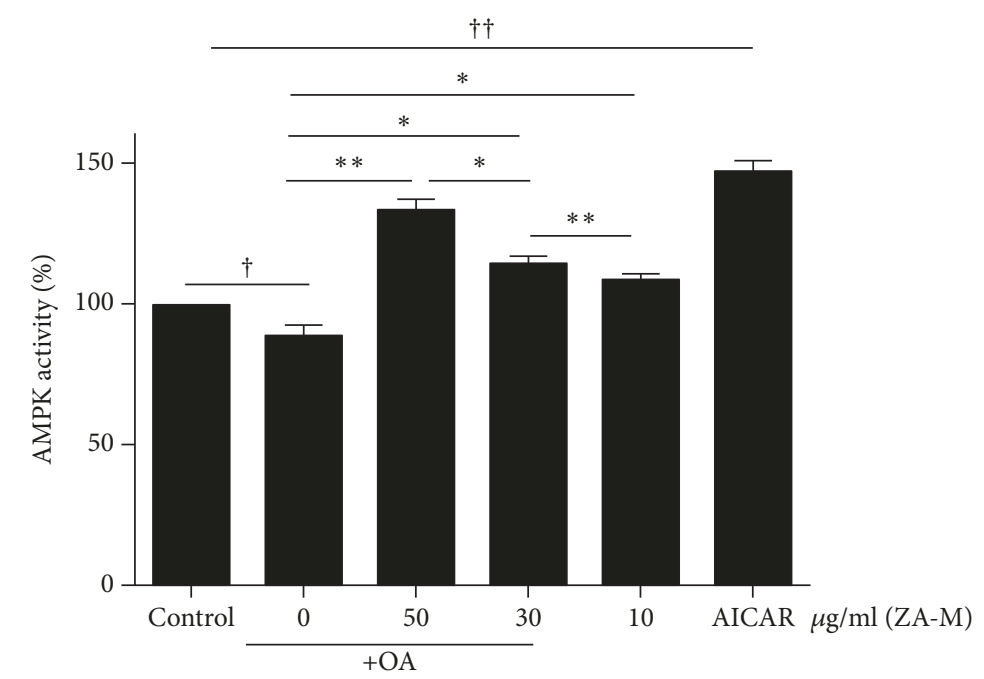

(c)

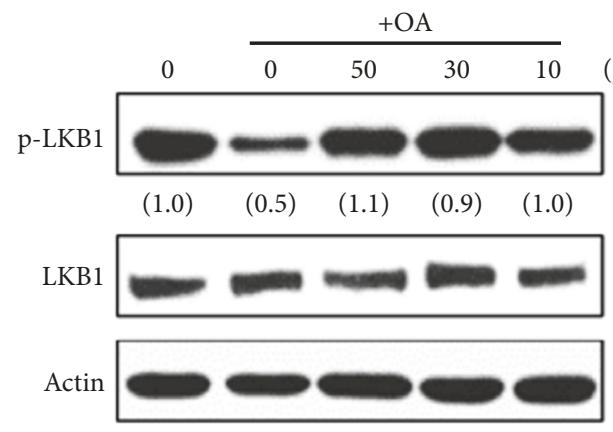

(d)

FIGURE 4: ZA-M activates the LKB1/AMPK signaling pathway. $(\mathrm{a}, \mathrm{b})$ Western blot analysis of phosphorylation status of AMPK (Thr 172) and ACC (Ser 79) after treatment of indicated concentrations of ZA-M (50, 30, and $10 \mu \mathrm{g} / \mathrm{ml})$ in the presence or absence of OA in HepG2 cells. (c) AMPK kinase activity. (d) Western blot analysis of phosphorylation status of LKB-1 after treatment of indicated concentrations of ZA-M $(50,30$, and $10 \mu \mathrm{g} / \mathrm{ml})$ in the presence of HepG2 cells. The bar graphs show the mean \pm SD of 3 independent experiments $\left({ }^{\dagger \dagger} p<0.01\right.$ and ${ }^{\dagger \dagger} p<0.001$ compared with the DMSO control; ${ }^{*} p<0.05$ compared with the OA treated control). 


\section{Supplementary Materials}

The original Western blot bands of Figures 3 and 4 . (Supplementary Materials)

\section{References}

[1] E.-B. Kwon, M.-J. Kang, S.-Y. Kim et al., "Zanthoxylum ailanthoides Suppresses Oleic Acid-Induced Lipid Accumulation through an Activation of LKB1/AMPK Pathway in HepG2 Cells," Evidence-Based Complementary and Alternative Medicine, vol. 2018, Article ID 3140267, 11 pages, 2018. 


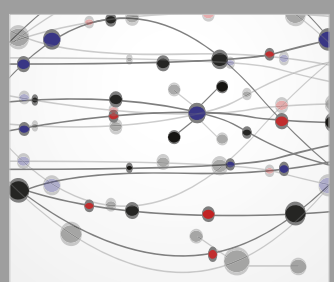

The Scientific World Journal
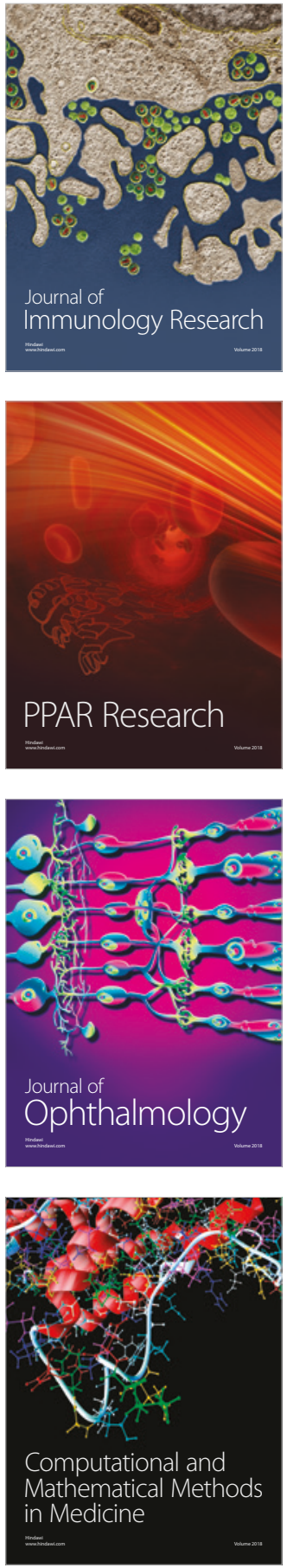

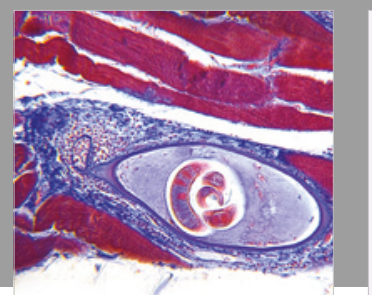

Gastroenterology Research and Practice

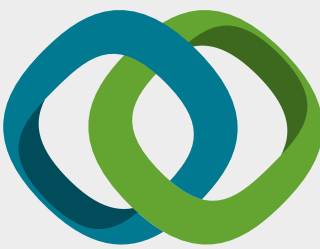

\section{Hindawi}

Submit your manuscripts at

www.hindawi.com
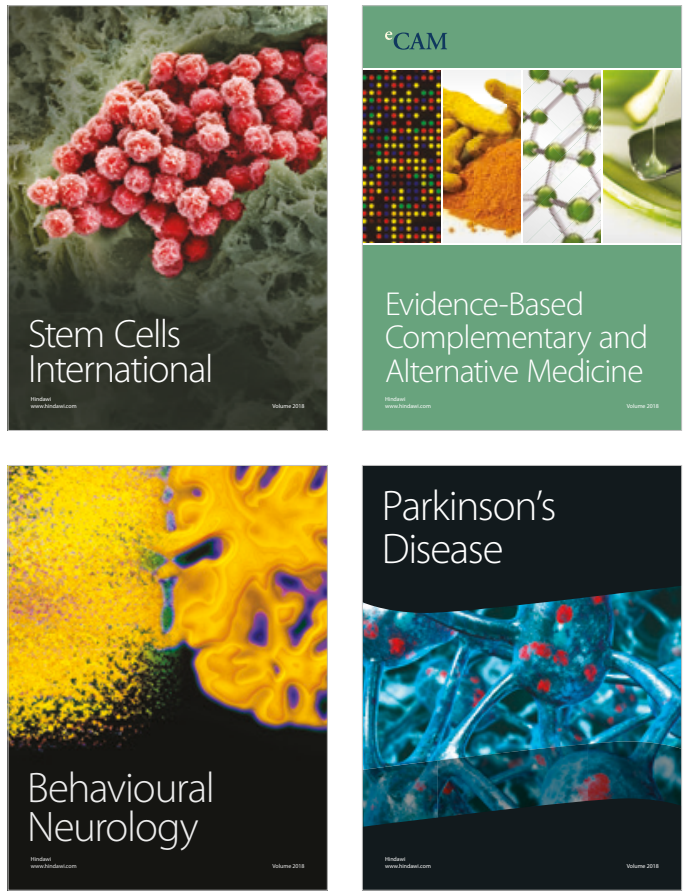

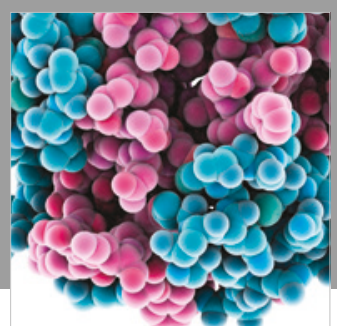

ournal of

Diabetes Research

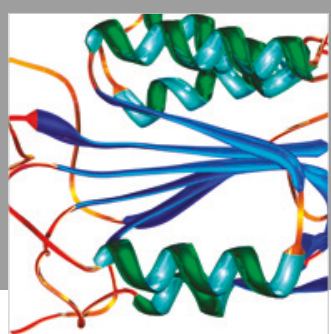

Disease Markers
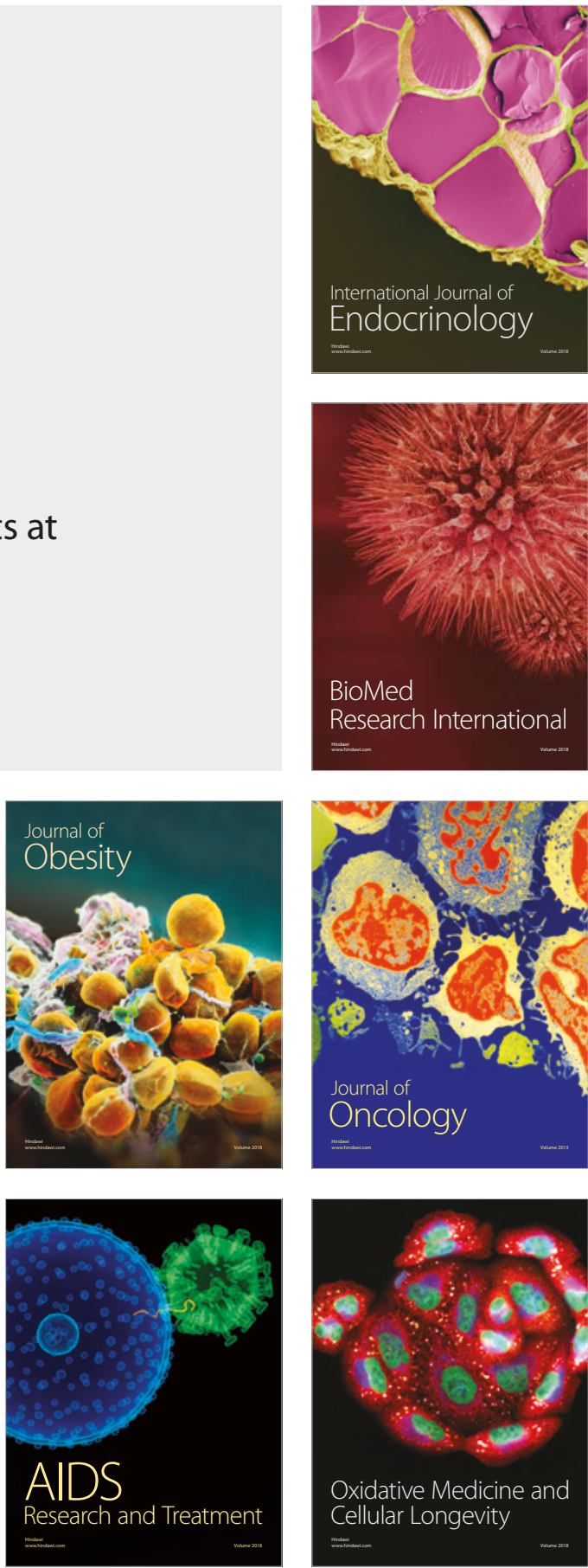